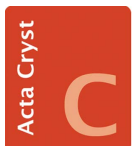

STRUCTURAL

CHEMISTRY

ISSN 2053-2296

Received 20 February 2020

Accepted 27 February 2020

Keywords: garnet; tolerance factor; crystal structure; phase stability

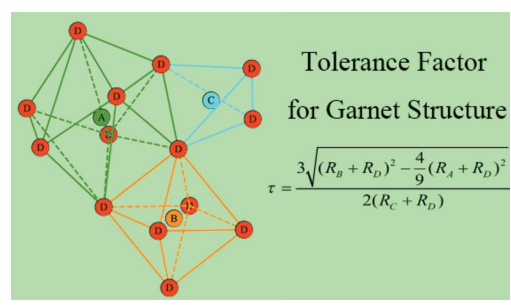

C 2020 International Union of Crystallography

\section{Tolerance factor and phase stability of the garnet structure. Corrigendum}

\author{
Zhen Song, Dandan Zhou and Quanlin Liu*
}

Beijing Key Laboratory for New Energy Materials and Technologies, School of Materials Science and Engineering, University of Science and Technology Beijing, Beijing 100083, People's Republic of China. ${ }^{*}$ Correspondence e-mail: qlliu@ustb.edu.cn

An error in an equation in the paper by Song et al. [Acta Cryst. (2019), C75, $1353-1358]$ is corrected.

In the last equation of $\$ 2.2$ (Expression for tolerance factor using ionic radius) of the paper by Song et al. (2019), there is a typographical error in the numerator, which should be $R_{A}+R_{D}$, not $R_{A}-R_{D}$. The correct equation is given below:

$$
\begin{aligned}
\tau & =\frac{L^{\prime}}{H}=\frac{2 \sqrt{\left(R_{B}+R_{D}\right)^{2}-\left(\frac{\sqrt{3}}{3} L\right)^{2}}}{\sqrt{\frac{2}{3}} \times \sqrt{\frac{8}{3}}\left(R_{C}+R_{D}\right)} \\
& =\frac{3 \sqrt{\left(R_{B}+R_{D}\right)^{2}-\frac{4}{9}\left(R_{A}+R_{D}\right)^{2}}}{2\left(R_{C}+R_{D}\right)} .
\end{aligned}
$$

\section{References}

Song, Z., Zhou, D. \& Liu, Q. (2019). Acta Cryst. C75, 1353-1358. 